\title{
Australian women's attitudes towards and understandings of the subdermal contraceptive implant: a qualitative study of never-users
}

\author{
Kumiyo Inoue, ${ }^{1,2}$ Marguerite Kelly, ${ }^{3,4}$ Alexandra Barratt, ${ }^{5}$ \\ Deborah Bateson, ${ }^{6,7}$ Alison Rutherford, ${ }^{8}$ Kirsten I Black, ${ }^{9}$ Mary Stewart, ${ }^{10}$ \\ Juliet Richters ${ }^{11}$
}

For numbered affiliations see end of article.

\section{Correspondence to}

Dr Kumiyo Inoue, School of Public Health and Community Medicine, University of New South Wales, Sydney 2052, Australia; k.inoue@student. unsw.edu.au

Received 21 October 2014 Revised 20 January 2016 Accepted 31 January 2016 Published Online First 3 March 2016

CrossMark

To cite: Inoue $K$, Kelly $M$, Barratt A, et al. J Fam Plann Reprod Health Care 2017:43:128-134.

\section{ABSTRACT}

Objective Few studies have explored Australian women's understandings of contraception. This study examined the attitudes towards, and understandings of, the subdermal contraceptive implant expressed by women living in New South Wales (NSW), Australia.

Study design As part of a larger qualitative study using in-depth, open-ended interviews in 2012-2013 with women aged 16-49 years who had ever used contraception ( $n=94), 65$ interviews from women who discussed or mentioned the subdermal implant, but had not previously used the device, were examined and analysed using thematic analysis.

Results The emergent themes were: satisfaction with current method; weak personal opinions and ambivalence; uncertainty due to specific concerns; and strong negative reactions - fear and dislike. Although there were a few positive perceptions expressed by women who had never used the subdermal implant, for the majority of women the perception was predominantly negative.

Discussion and conclusion Women tended to form negative impressions from the stories of other women about the subdermal implant. Interventions to enhance evidence-informed awareness of the relative advantages and disadvantages of the implant - for example, improved access to supportive contraceptive counselling - need investigation in the Australian context. Avenues to improve women's perceived control over the device could also be usefully investigated.

\section{INTRODUCTION}

It has been well documented internationally that long-acting reversible contraceptive (LARC) methods including the

\section{Key message points}

- Women's general awareness of the implant was high but some formed negative opinions about it on the basis of stories from other women.

- Because they had to rely on a health care practitioner for insertion and removal, women felt a lack of control in relation to the implant.

- Side effects such as bleeding, mood changes and weight gain were influential in forming negative opinions.

subdermal implant reduce unintended pregnancy and abortion rates. ${ }^{1}{ }^{2}$ Awareness amongst health professionals and women in Australia appears to be increasing, but policy directives are pending and the contraceptive pill remains the most commonly prescribed method of contraception in Australia. ${ }^{3} 4$ Contraception in Australia is primarily provided by general practitioners (GPs) working in private practice, with smaller numbers of women accessing contraception through government-funded or private family planning clinics, private gynaecologists or publicly funded outpatient clinics.

Use of the subdermal implant (hereafter referred to as 'the implant') in Australia has increased over the last decade $^{4}$ but is still lower than in some other comparable countries. ${ }^{5}$ Possible reasons for this, pointed out by some Australian clinicians at the community, 
health care provider and policy level, include lack of awareness of the method and continuing misinformation and misperceptions about it amongst potentially eligible users. ${ }^{6}$

Studies of implant use in Australia to date have been predominantly user-focused and clinical, ${ }^{7-12}$ and thus women's understandings and experiences have been understood largely in terms of those side effects and reasons for (dis)continuation that were considered by researchers to be clinically relevant. ${ }^{13}$

Studies reporting women's awareness of a range of methods are surprisingly scarce in developed countries. There have been few studies exclusively reporting never-users' understandings of the subdermal implant but more often, in studies to date, the subdermal implant has been grouped together with other LARCs. ${ }^{14-19}$ The target age group of women in these studies tends to be young. ${ }^{16-19}$ Awareness of the subdermal implant varied between studies, ${ }^{14-17}$ which is understandable given the timeframes, geographic locations, sample collections and target populations. Generally speaking, women who attend a community family planning clinic have a higher awareness of LARCs than those who attend GPs. ${ }^{15}$ All LARCs, including the implant, require the intervention of a health care professional for insertion and removal, and this was identified as an obstacle to promoting these methods. ${ }^{14}$

A qualitative study conducted in New Zealand reported women's lack of knowledge of the implant and suggested that this was due to its relatively recent introduction as a contraceptive method. ${ }^{16}$ A US survey found that only $8 \%$ of women had heard of the implant, and concluded that women who knew little about the method tended to be concerned about using a contraceptive that was new to them. ${ }^{17}$ In Okpo et al.'s qualitative study in Scotland, the implant was spontaneously mentioned by quite a number of women, referring to it as the implant or the 'rod'. The authors noted that negative testimonies often discouraged women from taking up the method. ${ }^{19}$

Bracken and Graham's ${ }^{18}$ online survey in the UK provides insight into women's feeling about having a foreign object in their body. The insertion of a foreign object into the arm was one of the notable disincentives to using the subdermal implant. Insertion and removal of the implant was identified as a 'disgusting' concept by the majority of participants in a study conducted in the UK. ${ }^{14}$ Clearly, the presence of a foreign object in the body can be an important issue for some women.

While quantitative data collected during clinical research studies provides some insight into why women have chosen the implant, the lack of qualitative data from women who have never used it has limited our understanding of why women choose not to use or even reject methods of contraception that a clinician may consider 'suitable' for them. It is important to hear from women who are not using this form of contraception.

This article reports Australian women's understandings of the subdermal implant and reasons for its non-use, based on an in-depth analysis of interviews with 65 women with experience of contraception.

\section{METHODS}

We adopted a descriptive qualitative approach that elucidates and interprets women's experiences, understandings and attitudes towards the implant.

\section{Data collection}

We collected the data as part of a wider study of women's contraceptive understandings and experiences (the CUE study) in which 94 in-depth openended interviews were carried out by four researchers between August 2012 and June 2013 in New South Wales (NSW). Women aged 16-49 years who had ever used contraception were eligible. Convenience sampling was the primary recruitment method. The majority of participants were recruited through Facebook and noticeboard advertisements at clinics, doctors' offices and university facilities. A small number of participants were recruited by snowballing sampling. Each participant read and signed a consent form at the time of her interview and received $A \$ 30$ as reimbursement for any expenses.

We encouraged women to speak openly and when women volunteered relevant stories, we used a simple open-ended questioning style including phrases such as: 'Then what happened?', 'Tell me more about that', 'What are your thoughts/feelings about that?' to explore their stories further. If the implant was not mentioned during the interview, a prompt was used, such as 'Have you ever heard about the subdermal implant (or bar/rod)? If so, tell me your thoughts about it'. Sometimes the brand name was used as a prompt.

\section{Inclusion and exclusion criteria}

Data from all women in our sample who discussed or mentioned the implant but who had never used this method $(n=65)$ were included in this analysis. Data about the experiences of 10 women who had used the implant and other hormonal methods are discussed in other papers.

\section{Data analysis}

The audio recorded interviews were transcribed verbatim by a professional transcriber, and then checked for accuracy and de-identified by the first two authors. Participants' names were replaced by pseudonyms.

The first two authors conducted an inductive thematic analysis following the phases outlined by Braun and Clarke. ${ }^{20}$ After immersing ourselves in the data by repeatedly listening to the interview recordings and 
rereading the transcripts, we systematically coded transcripts line by line to identify patterns in the data. During coding and data analysis, questions such as 'What is going on?', 'What does this expression mean?', 'Why did she say this?' and 'What does this mean to her?' were constantly pondered. The aim of our thematic analysis was not to categorise women into a typology or extract factors that discourage or encourage uptake of the implant. Primarily, we aimed to explore women's understandings of the implant and their perceptions of its viability for their body. To this end, we listened carefully to the language and expressions used by women during interviews. Themes were derived by analysing not only women's descriptions but also how they expressed them. The four themes do not exist in isolation, nor do they define the women themselves. Frequently, more than one theme was evident in the perceptions of a single woman.

Qualitative data analysis software (NVivo $10^{\mathrm{TM}}$, QSR International) was used to highlight and organise codes. Defining and renaming codes, and considering how concepts related to each other as well as the broader dataset, resulted in final themes and subthemes being identified. 'The rod' and the brand name 'Implanon' were often used by women to describe the implant. (Only one brand of implant is currently available in the Australian market.)

\section{Ethical approval}

The study was approved by the Human Research Ethics Committee of the University of New South Wales (UNSW) (HC11504) and ratified by Family Planning NSW and University of Sydney ethics committees.

\section{RESULTS AND DISCUSSION}

A total of 65 women who had never used the implant reported their thoughts and opinions about it. These women ranged in age from their late teens to their late $40 \mathrm{~s}$, and lived in urban, rural and regional areas. More than half of the women were in their 20s, and nearly a quarter were in their 30 s. Some women in their 40s gained their knowledge of the implant through their daughters' experiences of using it. Demographic information about these women is found in Table 1.

\section{Understandings and attitudes of women}

We identified the following four major themes in the data from women who had never used the implant: (1) satisfaction with current method; (2) noncommittal responses; (3) uncertainty; and (4) fear and dislike.

Theme 1: Satisfaction with current method

Some women simply saw no need to change from a current method that suited them. As Ava, in her 20s,
Table 1 Demographics of the 65 women who discussed the implant

\begin{tabular}{lr}
\hline Characteristic & $\boldsymbol{n}$ \\
\hline Age group (years) & 5 \\
16-19 & 36 \\
$20-29$ & 15 \\
$30-39$ & 9 \\
$40-49$ & \\
Ethnic background" & 51 \\
$\quad$ Anglo-Celtic & 14 \\
Asian, Middle Eastern, African, Indigenous & 33 \\
Locationt & 16 \\
$\quad$ Urban & 14 \\
Regional & 2 \\
Rural & 38 \\
Urban and rural & 27 \\
Relationship status & \\
$\quad$ In a relationship & \\
Not in a relationship & \\
\hline *As described by women themselves. \\
tUrban' refers to the state capital, Sydney; 'regional' refers to other large \\
towns and cities, and 'rural' refers to smaller rural towns and localities.
\end{tabular}

said, "I am happy with the pill, so I don't want to disturb something that I'm happy with".

Other women who expressed their clear reasons for using the pill in terms of improving a skin condition and/or controlling their menstrual cycle were not interested in the implant as they did not perceive it to have similar benefits. Some women did not like hormonal contraceptives at all and therefore preferred other methods, especially the condom. Women who preferred the hormone-releasing intrauterine device (IUD) over the implant discussed the benefits of experiencing fewer issues with bleeding and more localised release of hormones with the hormonereleasing IUD. Issues associated with the hormones in the implant were referred to by some women. Overall, satisfaction with other contraceptive methods and/or perceived benefits of other methods meant that some women were not interested in trying the implant.

Theme 2: Noncommittal responses

This theme is constituted by 'low affect' accounts that displayed only a basic awareness or very limited understanding of the implant (i.e. the name only or awareness of some features) as well as accounts containing theoretical knowledge rather than personal opinion. These accounts describe the implant as good or bad, suitable or unsuitable, without giving detailed reasons. Some women used general expressions such as "I am aware of" or "I have heard about it", and rarely expanded on their own opinion.

A few women expressed weakly positive or indifferent perceptions, mainly because they had not heard any bad reports about the method. The specific 
positive characteristics mentioned briefly by some women were reliability (very high rate of protection from pregnancy), affordability (relatively cheap) and convenience (long-lasting and not requiring daily action).

Some respondents, particularly women employed in the health care sector, did not base their views on the limited reports of others. Instead of expressing their personal opinions and feelings they tended to provide general understandings, quite possibly gained from their work. These included their perception of the types of women who would be ideal candidates for the implant, such as young women and women who do not remember to take the pill.

Theme 3: Uncertainty

Some women who expressed uncertainty about the implant were hesitant to use it due to specific concerns, including: a perception that little is known about the method and its long-term effects; an assumption that it would be expensive; the possible short-term side effects; a perception that evidence for the safety of the hormone in the implant is lacking; and the possible negative effects of the method on fertility in the longer term. Some women who expressed uncertainty also admitted that it seemed to be a good method. A number of women reported that even though they had heard some positive stories about the implant, the negative ones led them to consider the method 'risky'.

Much of the uncertainty expressed by women related to the perception that the implant is a relatively new method, causing them to question what their own experience might be if they were to try it.

\section{"... or get that thing in your arm, the rod. But I just, I know they're so new I don't wanna try them 'cause they're just so new. I just, just in case there's a side effect that no one really knows about". [Victoria, in her 30s]}

Women felt uncertain about the implant because they perceived it as a product in its infancy. The possibility of menstruation ceasing as a side effect of using the implant was a concern for some women, who feared the possibility of pregnancy being masked or future fertility being affected.

\begin{abstract}
"Sometimes it's a relief when I get my period, yes, but just like it's just how we were made. I don't know. Like I just find it really bizarre to be messing with that and like I've, I just get scared that like if you don't have your periods for whatever long, like it'll mess up fertility later on in life. Not so much with the pill but more with that rod. I don't know ..." [Amelia, in her 20s]
\end{abstract}

Theme 4: Fear and dislike

Some women had strong negative reactions, using words such as 'gross', 'weird', 'yuk', 'bizarre' and 'creepy' based on hearing about the implant from others or reading about it. Quite often, a response such as "I just don't like the idea of it" was offered when further clarification was sought. A few women gave a more detailed explanation of their feelings. One woman described the concept of having an implant as akin to being 'microchipped' and another described it as being 'tracked'.

"I almost feel it. Like, when I say 'Implanon', I'm stroking my arm ... I kind of I feel like a, you're being microchipped or something. [Oh] Yeah. I think it's amazing technology and that [Yeah] but I just, I don't think I could do it." [Maya, in her 20s]

The experience of viewing or touching the implant in another woman's arm tended to have a negative impact on women's impressions. Fear about the placement of the implant was exacerbated for some women by a lack of understanding about it.

"It just does not feel right, you know like, like a cheap kind of thing? A little rod? How big and like how deep inside my arm? ... and it cannot travel anywhere else?" [Desiree, in her 30s]

Some women expressed strong negative reactions with clear reasons, such as dislike of the possibility of irregular bleeding and of mood changes, fear of the long-term impact on fertility, and fear of painful insertion and removal procedures. Other women disliked that a level of personal control would be taken from them if they used the implant because they would have to access a doctor for insertion and removal. Women's reasons for disliking the implant often emerged as the interview progressed. Furthermore, women's reasons were sometimes multilayered. Layla started with an account of her own squeamishness and then proceeded to discuss a practical issue relating to knowledge about pregnancy.

"Personally, I wouldn't want the rod that my friends
get because I'm really icky when it comes to like veins
and blood, and all that. And [if] they really have to get
it out, it would just make me sick. And also, with the
rod, they, the girls tell me that sometimes they don't
get a period at all for 2 or 3 years so how do you
know if that's $1 \%$ that you've fallen pregnant or that
$0.5 \%$, or whatever it is?" [Layla, in her 20s].

\section{DISCUSSION}

Although the larger study that included these interviews did not aim to quantitatively examine a feature of Australian women in regard to their understandings of the implant, it was notable that more than two-thirds of the 94 women had some awareness of it, and 10 women had ever used the implant. Awareness of the implant, however, does not necessarily correlate with comprehensive knowledge, and this was evident in a number of accounts from women who displayed their uncertainty and even ambivalence. The implant was introduced to the Australian 
market in 2001 but women's perception that it is a new method still prevails. Overall, never-users' perceptions of the implant were negative. Furthermore, awareness of the positive characteristics of the implant was often overridden by negative or inconsistent stories of the implant heard from other women, such as stories about bleeding irregularity and discontinuation experiences.

Australian women feel they lack information about contraceptives. ${ }^{21}$ We found family, friends and doctors were influential and women largely formed their opinions based on stories they had heard. This is in line with other study findings. ${ }^{10} 12$ 14-16 1922 Based on an analysis of audio recorded consultations, Levy et al. ${ }^{22}$ reported that social influences may not be recognised by doctors during contraceptive consultations but that they are an important factor to consider when understanding women's contraceptive preferences.

Interestingly, participants who were health care workers tended to focus the discussion of the implant on which women would be ideal candidates for the method, rather than expressing their personal opinions about it and its suitability for them personally. The limited number of accounts does not enable us to make firm conclusions about this but it is possible to speculate that some level of ambivalence exists, and that women who are health care workers may be inclined to recommend the method to others, while at the same time feeling some reluctance about using it themselves.

Extreme negative expressions and even refusal to consider the implant were voiced by women who were satisfied with their current method or who had strong negative reactions such as fear and dislike. These were largely women who were well informed about the non-contraceptive benefits of the oral contraceptive or who preferred non-hormonal contraceptives. Although one group accepted hormones and the other did not, their shared perceptions related to concerns about the influence of introduced hormones on the body. Over and above concerns about hormones, the most commonly expressed concern related to troublesome vaginal bleeding, and this has also been reported in other studies. ${ }^{10-12}$ Even accounts from implant users that their bleeding had settled down over time did not tend to change the negative opinions of never-users. Clearly, the way in which bleeding patterns are discussed and managed affects some women's attitudes.

Another aspect of implant use that was most disliked was the idea of the implant being inserted under the skin, with many women perceiving the procedure as quite invasive. This is consistent with findings reported by Bracken and Graham, ${ }^{18}$ but our findings add a further insight related to the medical procedure for insertion. Because they could not start or stop using the implant without the involvement of a health care professional, some women felt that the method was less user-controlled than other methods such as the pill and the condom. Unlike users of the implant, ${ }^{23}$ never-users in our study liked to have a high level of individual control over the uptake and discontinuation of contraceptives. This argument can extend to the concept of reversibility. Reviews of literature on clinical trials indicate that the implant is a 'highly reversible' contraceptive method ${ }^{24} 25$ and this is often stressed in educational materials. However, this understanding is not reflected in the perceptions of women who have never used it. This gap between women's perceptions and literature about the implant may be related to different definitions of reversibility. Although the effects of the implant can be reversed by its removal, women seem to value having control over the cessation of the method. Similarly, Okpo et al. ${ }^{19}$ found that women in their study perceived the term 'reversible' negatively, because they understood it to involve medical intervention and therefore a lack of user control over when and where to stop their chosen method.

\section{LIMITATIONS AND FURTHER RESEARCH}

Many of the participants in the larger study had nothing to say about the implant, either positive or negative, and thus their voices do not appear in this paper. Additional investigation of the entire data set of the CUE study using selective coding would allow us to further interpret women's understandings about contraceptives. Our sample included respondents from a wide range of backgrounds, including young mothers and women from regional and rural areas as well as tertiary educated urban residents. However, the purpose of this was to hear a range of voices, not to achieve a generalisable sample in the statistical sense.

\section{CONCLUSIONS AND RECOMMENDATIONS}

The understandings expressed by women who had never used the implant tended to be either indifferent or negative. Many of the women had formed an opinion without having detailed information about the implant and how it works in the body. One of the clinical implications of our findings is the need for dissemination of evidence-based information to women in the community, in order to raise the awareness of women who would potentially benefit from using the implant, but who appear to reject it based on hearsay.

Our study suggests that many negative perceptions of the implant expressed by women relate partly to its design and insertion (implantation of a palpable rod in the arm involving an incision and possible pain), as well as to the perceived short- and long-term side effects. Clinicians with a greater understanding of women's perceptions of the implant may be able to provide better, more targeted counselling about its 
relative advantages and disadvantages, to support informed contraceptive choice.

Although women cannot insert the implant themselves, providing as much autonomy in decision making as possible regarding the timing of insertion and removal of the device, and offering supportive counselling may be helpful for women who experience difficulty with an aspect of the device.

Furthermore, lack of information or awareness limits women's freedom of choice across methods. Avenues must be made available for women to develop up-to-date knowledge of contraceptives so that they are in a position to make well-informed choices. Proactive attitudes also need to be nurtured during early sex education so that women will be motivated and empowered to become and remain informed.

In Australia, prescription medications cannot be advertised publicly. ${ }^{26}$ A subsequent lack of popular knowledge may explain why hormonal contraceptive methods tend not to be subjects of popular discourse or public discussion. Women's opinions about different contraceptive methods are complex and multidimensional, resulting from a combination of vicarious learning from others, limited contraceptive information, personal experience, and personal perceptions of method suitability. If women are to consider the implant as a viable option, interventions need to target the spectrum of women's understandings and concerns, as well as the sources of their information.

\author{
Author affiliations \\ ${ }^{1} \mathrm{PhD}$ Scholar, School of Public Health and \\ Community Medicine, University of New South \\ Wales, Sydney, Australia \\ ${ }^{2}$ Adjunct Senior Researcher, School of Health \\ Sciences, University of Tasmania, Hobart, Australia \\ ${ }^{3}$ Project Officer, Western Sydney University, Sydney, \\ Australia \\ ${ }^{4}$ Project Officer, School of Public Health and \\ Community Medicine, University of New South \\ Wales, Sydney, Australia \\ ${ }^{5}$ Professor, Sydney School of Public Health, University \\ of Sydney, Sydney, Australia \\ ${ }^{6}$ Medical Director, Family Planning New South Wales, \\ Ashfield, Australia \\ ${ }^{7}$ Clinical Associate Professor, Obstetrics, Gynaecology \\ and Neonatology, University of Sydney, Sydney, \\ Australia \\ ${ }^{8}$ Senior Lecturer (Conjoint), University of New South \\ Wales, Sydney, Australia \\ ${ }^{9}$ Associate Professor (Obstetrics, Gynaecology and \\ Neonatology), Central Clinical School, University of \\ Sydney, Sydney, Australia \\ ${ }^{10}$ Senior Medical Officer: Research and Education, \\ Family Planning New South Wales, Ashfield, Australia \\ ${ }^{11}$ Professor, School of Public Health and Community \\ Medicine, University of New South Wales, Sydney, \\ Australia
}

Acknowledgements The authors gratefully acknowledge the contributions of the study participants. This Australian Research Council Linkage Project was in partnership with Family Planning NSW and the University of Sydney.

Funding Australian Research Council linkage grant (LP110200996).

Competing interests Deborah Bateson and Mary Stewart are employed by Family Planning NSW, which conducts clinical trials sponsored by pharmaceutical companies. Their organisation receives fees from MSD for contraceptive implant training and sponsorship from Bayer Healthcare for intrauterine device training sessions. Deborah Bateson has received individual support to attend national and international conferences from MSD and Bayer Healthcare. Kirsten Black was a trainer on the implant insertion programme supported by MSD. She was a consultant on an international advisory board for Bayer Healthcare and has received individual support to attend a conference as a presenter.

Ethics approval Human Research Ethics Committee, University of New South Wales.

Provenance and peer review Not commissioned; externally peer reviewed.

\section{REFERENCES}

1 Family Planning NSW. Reproductive and Sexual Health in Australia. 2013. http://www.fpnsw.org.au/ rshinaust_book_webedition_1.pdf [accessed 26 October 2015].

2 Bateson D, Harvey C, McNamee K. Contraception: An Australian Clinical Practice Handbook (3rd edn). Family Planning New South Wales, Family Planning Queensland and Family Planning Victoria, 2012.

3 Mazza D, Harrison C, Taft A, et al. Current contraceptive management in Australian general practice: an analysis of BEACH data. Med J Aust 2012;197:110-114.

4 Richters J. The Australian Study of Health and Relationships: Contraceptive choices. Invited presentation at the RCOG World Congress 2015, Royal College of Obstetricians and Gynaecologists (RCOG) and Royal Australian and New Zealand College of Obstetricians and Gynaecologists (RANZCOG), Brisbane, 15 April 2015. Abstract in Section C22: Sexual Health, p. 116 of conference program, New endeavours in women's health. www.rog2015.com.

5 Sexual Health and Family Planning Australia. Time for a Change: Increasing the Use of Long Acting Reversible Contraceptive Methods in Australia. 2013. http://www.fpv.org.au/assets/ LARCstatementSHFPAFINAL.pdf [accessed 26 October 2015].

6 Black KI, Bateson D, Harvey C. Australian women need increased access to long-acting reversible contraception. Med J Aust 2013;199:317-318.

7 Weisberg E, Fraser I. Australian women's experiences with Implanon. Aust Fam Phy 2005;34:694-696.

8 Weisberg E, Hickey M, Palmer D, et al. A pilot study to assess the effect of three short-term treatments on frequent and/or prolonged bleeding compared to placebo in women using Implanon. Hum Reprod 2006;21:295-302.

9 Harvey C, Seib C, Lucke J. Continuation rates and reasons for removal among Implanon users accessing two family planning clinics in Queensland, Australia. Contraception 2009;80:527-532.

10 Wong RC, Bell RJ, Thunuguntla K, et al. Implanon users are less likely to be satisfied with their contraception after 6 months than IUD users. Contraception 2009;80:452-456.

11 Lewis LN, Doherty DA, Hickey M, et al. Implanon as a contraceptive choice for teenage mothers: a comparison of contraceptive choices, acceptability and repeat pregnancy. Contraception 2010;81:421-426. 
12 Weisberg E, Bateson D, McGeechan K, et al. A three-year comparative study of continuation rates, bleeding patterns and satisfaction in Australian women using a subdermal contraceptive implant or progestogen releasing intrauterine system. Eur J Contracept Reprod Health Care 2014;19:5-14.

13 Inoue K, Barratt A, Richters J. Does research into contraceptive method discontinuation address women's own reasons? A critical review. J Fam Plann Reprod Health Care 2015;41:292-299.

14 Glasier A, Scorer J, Bigrigg A. Attitudes of women in Scotland to contraception: a qualitative study to explore the acceptability of long-acting methods. J Fam Plann Reprod Health Care 2008;34:213-217.

15 Mansour D, Rosales C, Cox M. Women's awareness of long acting, reversible contraceptive methods (LARCs) in community family planning clinics and general practice. Eur J Contracept Reprod Health Care 2008;13:396-399.

16 Rose SB, Cooper AJ, Baker NK, et al. Attitudes toward long-acting reversible contraception among young women seeking abortion. J Womens Health 2011;20:1729-1735.

17 Spies E, Askelson NM, Gelman E, et al. Young women's knowledge, attitudes, and behaviours related to long-acting reversible contraceptives. Womens Health Issues 2010;20:394-399.

18 Bracken J, Graham CA. Young women's attitudes towards, and experiences of, long-acting reversible contraceptives. Eur J Contracept Reprod Health Care 2014;19:276-284.
19 Okpo E, Allerton L, Brechin S. 'But you can't reverse a hysterectomy! Perception of long acting reversible contraception (LARC) among young women aged 16-24 years: a qualitative study. Public Health 2014;128:934-939.

20 Braun V, Clarke V. Using thematic analysis in psychology. Qual Res Psychol 2006;3:77-101.

21 Dixon SC, Herbert DL, Loxton D, et al. 'As many options as there are, there are just not enough for me': contraceptive use and barriers to access among Australian women. Eur J Contracept Reprod Health Care 2014;19:340-351.

22 Levy K, Minnis AM, Lahiff M, et al. Bringing patients' social context into the examination room: an investigation of the discussion of social influence during contraceptive counseling. Womens Health Issues 2015;25:13-21.

23 Darney P, Patel A, Rosen K, et al. Safety and efficacy of a single-rod etonogestrel implant (Implanon): results from 11 international clinical trials. Fertil Steril 2009;91:1646-1653.

24 Graesslin O, Korver T. The contraceptive efficacy of Implanon ${ }^{\circledR}$ : a review of clinical trials and marketing experience. Eur J Contracept Reprod Health Care 2008;13:4-12.

25 Adams K, Beal MW. Implanon: a review of the literature with recommendations for clinical management. J Midwifery Womens Health 2009;54:142-149.

26 Australian Government. Department of Health. Regulation of Therapeutic Goods Advertising in Australia. https://www.tga. gov.au/regulation-therapeutic-goods-advertising-australia [accessed 26 October 2015].

\section{FSRH Members' Enquiries meet new research - avoiding needles as a barrier to implant insertion}

One of the barriers to the use of long-acting reversible contraception identified in an article in this journal issue is negative feelings about having an implant inserted below the skin and fear of painful insertion and removal procedures $^{1}$ - a challenge also raised by a FSRH Members' Enquiry about alternative approaches to skin analgesia for implant insertion. ${ }^{2}$

In this issue, Inoue et al.'s qualitative study of Australian women's attitudes to and understanding of the contraceptive implant in never-users ${ }^{1}$ finds negative perceptions of the method. As well as being put off by bleeding, women described negative feelings about having an implant inserted below the skin and fear of painful insertion and removal procedures. The authors emphasise the importance of 'story', and of autonomy in decision-making.

In FSRH Members' Enquiry https://www.fsrh.org/documents/ceu-edme0028-feb2015/, the clinician asks whether cryogesic spray can be used when removing implants. The Clinical Effectiveness Unit (CEU) responds by describing a small study that used ethyl chloride spray as anaesthesia for implant insertion, with high satisfaction for both patients and clinicians, and less reported bleeding and bruising. The CEU also cites anecdotal evidence of ethyl chloride's use for implant removals. The approach the CEU describe in their evidence review shows potential for addressing the needs of women afraid of needles, and may provide another option to offer women, enhancing 'autonomy in decision-making'.

\section{Susanna Hall}

Trainee Representative, Journal of Family Planning and Reproductive Health Care, and Specialty Trainee in Community Sexual and Reproductive Healthcare, Bristol Sexual Health Service, Bristol, UK; susanna.hall@uhbristol.nhs.uk

\section{References}

1 Inoue K, Kelly M, Barratt $A$, et al. Australian women's attitudes towards and understandings of the subdermal contraceptive implant: a qualitative study of never-users. J Fam Plann Reprod Health Care 2017;43:128-134.

2 FSRH Member's Enquiry Response (Ref. EdME0028). https://www.fsrh.org/documents/ceu-edme0028-feb2015/ [accessed 13 February 2017]. 